\title{
A NOTE ON CONTINUITY OF SEMIGROUPS OF MAPS
}

\author{
PAUL R. CHERNOFF 1
}

ABSTRACT. An example is given of a separately continuous semigroup of transformations on Hilbert space which fails to be jointly continuous at $t=0$.

1. Let $X$ be a topological space and $\left\{F_{t}: t \geq 0\right\}$ a one-parameter semigroup of continuous maps of $X$ into itself; that is, $F_{s+t}:=F_{s} \circ F_{t}$ and $F_{0}=$ identity. Suppose also that for each $x$ in $X$ the mapping $t \mapsto F_{t}(x)$ is continuous. Thus $(t, x) \mapsto F_{t}(x)$ is continuous in each variable separately. Under additional hypotheses we can conclude that this map is jointly continuous. Specifically, in [1] it is shown that if $X$ is metrizable, then every point $(t, x)$ with $t>0$ is a point of joint continuity. Moreover, Dorroh [2] has shown that if $X$ is locally compact and $\sigma$-compact then every point $(t, x)$, including points with $t=0$, is a point of joint continuity.

On the other hand, it was stated in [1] that there is an example for which joint continuity fails at $t=0$, with $X$ a certain subset of $R^{2}$. (There is a misprint in [1] on p. 1046; it is erroneously stated that such an example exists with $X=R^{2}$. This is, of course, ruled out by Dorroh's result.)

The aim of this note is to present an example illustrating failure of joint continuity at $t=0$ with $X$ a Hilbert space. Thus Dorroh's result does not generalize from finite-dimensional to infinite-dimensional manifolds.

2. Before giving the construction, it seems worthwhile to present a proof of joint continuity at $t=0$ which is substantially more elementary than Dorroh's argument. We shall assume that the space $X$ is locally compact and metrizable (rather than locally compact and $\sigma$-compact as in [2]).

Suppose that $\left\{F_{t}: t \geq 0\right\}$ is a separately continuous semigroup of maps on $X$. By [1] we have joint continuity for $t>0$. We must establish joint continuity at $t=0$. That is, given $x$ in $X$ and sequences $x_{n} \rightarrow x, t_{n} \rightarrow 0$, we have to show that $F_{t_{n}}\left(x_{n}\right) \rightarrow x$. If this is not the case, then there is a compact neighborhood $K$ of $x$ such that $F_{t_{n}}\left(x_{n}\right) \notin K$ for arbitrarily large $n$. Since $x_{n} \rightarrow x$, we may as well assume that $x_{n} \in K$, but $F_{t_{n}}\left(x_{n}\right) \notin K$, for all $n$. But then, because $F_{t}\left(x_{n}\right)$ is continuous in $t$, a connectedness argument

Received by the editors December 14, 1974 .

AMS (MOS) subject classifications (1970). Primary 20M20, 541115; Secondary $57 \mathrm{~A} 20$.

1 Research partially supported by NSF grant GP-30798X. 
shows that $F_{s_{n}}\left(x_{n}\right)$ lies on the boundary $B$ of $K$ for some $s_{n}$ between 0 and $t_{n}$. Since $B$ is compact, we may assume that $F_{s_{n}}\left(x_{n}\right)$ converges to a point $y$ in $B$. Now obsetve that if $t>0$,

$$
F_{t}(y)=\lim F_{t} F_{s_{n}}\left(x_{n}\right)=\lim F_{t+s_{n}}\left(x_{n}\right)=F_{t}(x),
$$

where joint continuity at $(t, x)$ is used at the last step. If we finally let $t$ converge to 0 in (1), we conclude that $y=x$. But this is a contradiction, since $x$ is in the interior of $K$.

3. To prepare the ground for the construction of the Hilbert space example, we will exhibit the example on the subset of $R^{2}$ which we mentioned above.

We define the subset $X$ as follows. Let $p$ be the point $(1,0)$. Introducing polar coordinates in the usual way, let $A=\{(r, \theta): r>1,0<\theta<2 \pi\}$. Then let $X=A \cup\{p\}$. (Thus $X$ is obtained from $R^{2}$ by deleting the positive $x$-axis and the closed unit disk, then restoring the point $p$. Note that $X$ is locally compact except at $p$.)

Define $h(\theta)=\theta /(2 \pi-\theta)$. The function $h$ maps the interval $(0,2 \pi)$ home omorphically onto $(0, \infty)$.

Now, for $t \geq 0$, define the map $F_{t}: X \rightarrow X$ in the following way. Set $F_{t}(p)=p$; and if $r>1$, put $F_{t}(r, \theta)=\left(r, h^{-1}[h(\theta)+t /(r-1)]\right)$. It is straightforward to check that $\left\{F_{t}: t \geq 0\right\}$ is a semigroup. (Roughly speaking, the flow $F_{t}$ makes the circular arc with radius $r>1$ collapse in a counterclockwise sense with increasing velocity as $r \rightarrow 1$.) To see that $F_{t}$ is continuous for fixed $t>0$, it is only necessary to worry about what happens at the point $p$. So let $x_{n}=\left(r_{n}, \theta_{n}\right)$ with $r_{n} \rightarrow 1, \theta_{n} \rightarrow 0$ or $2 \pi$. Then $h\left(\theta_{n}\right)+t /\left(r_{n}-1\right) \rightarrow \infty$, and so $h^{-1}\left[h\left(\theta_{n}\right)^{n}+t /\left(r_{n}-1\right)\right] \rightarrow 2 \pi$. Hence, $F_{t}\left(r_{n}, \theta_{n}\right) \rightarrow(1,2 \pi)^{n}=p=F_{t}(p)$. If $t=0, F_{t}=$ identity. Also, it is clear that $F_{t}(x)$ is continuous in $t$ for fixed $x$ in $X$.

Finally, we verify the failure of joint continuity at $p$ with $t=0$. Take $x_{n}=\left(r_{n}, \theta_{n}\right)$ with $r_{n}=1+1 / n$ and $\theta_{n}=1 / n$. Take $t_{n}=1 / n$. Then $x_{n} \rightarrow p$ and $t_{n} \rightarrow 0$, but

$$
F_{t}\left(x_{n}\right)=\left(r_{n}, h^{-1}[h(1 / n)+1]\right) \rightarrow\left(1, h^{-1}(1)\right)=(1, \pi) \neq p .
$$

We can now obtain an example on an open subset of Hilbert space quite cheaply (modulo infinite-dimensional topology!). Indeed, let $H$ be a separable, infinite-dimensional Hilbert space. The space $X$ considered above is easily seen to be a locally finite-dimensional simplicial complex; that is, the two-dimensional space $X$ can be triangulated and homeomorphically embedded as a piecewise linear subset of $H$ so that the vertices of the triangulation 
correspond to mutually orthogonal unit vectors. Hence, by [5, Theorem 3], the product space $X \times H$ is a manifold modelled on $H$. By the results in [3], $X \times H$ is homeomorphic to an open subset of $H$. We then simply take as our semigroup on $X \times H$ the maps $G_{t}=F_{t} \times I$.

The referee has observed that a simple modification of this construction yields a semigroup acting on the whole space $H$. Consider the metric cone $C$ of $X:$ if $X$ is embedded as a piecewise linear subset of $H$, then $C$ is the subset of $H \times \mathbf{R}$ consisting of the points $(\lambda x, 1-\lambda)$ with $x \in X$ and $0 \leq$ $\lambda \leq 1$. The semigroup $F_{t}$ extends in the obvious way to $C$ : we define $F_{t}^{\prime}(\lambda x, 1-\lambda)=\left(\lambda F_{t}(x), 1-\lambda\right)$. Then $F_{t}^{\prime} \times I$ is the desired semigroup on $C \times H$. The point is that $C$ is a contractible locally finite-dimensional simplicial complex, and so, by [3, Corollary 3], $C \times H$ is homeomorphic to $H$.

Acknowledgement. I wish to thank Professor Victor Klee for some interesting correspondence concerning the proof that $X \times H$ is homeomorphic to an open subset of $H$. In particular, Klee has shown that this can be obtained via the machinery developed in [4].

\section{REFERENCES}

1. P. R. Chernoff and J. Marsden, $O_{n}$ continuity and smoothness of group ac= tions, Bull. Amer. Math. Soc. 76 (1970), 1044-1049. MR $42 \# 419$.

2. J. R. Dorroh, Semi-groups of maps in a locally compact space, Canad. J. Math. 19 (1967), 688-696. MR 37 \#4777.

3. D. W. Henderson, Infinite-dimensional manifolds are open subsets of Hilbert space, Topology 9 (1970), 25-33. MR 40 \#3581; erratum, 40, p. 1706.

4. V. L. Klee, Jr., Convex bodies and periodic homeomorphisms in Hilbert space, Trans. Amer. Math. Soc. 74 (1953), 10-43. MR 14, 989.

5. J. E. West, Products of complexes and Fréchet spaces which are manifolds, Trans. Amer. Math. Soc. 166 (1972), 317-337. MR $45 \# 2756$.

DEPARTMENT OF MATHEMATICS, UNIVERSITY OF CALIFORNIA, BERKELEY。 CALIFORNIA 94720 